\title{
Efficient Analysis of Structures with Rotatable Elements Using Model Order Reduction
}

\author{
Grzegorz FOTYGA, Krzysztof NYKA \\ Department of Electronics, Telecommunication, and Informatics, \\ Gdansk University of Technology \\ Narutowicza 11/12, 80-233 Gdansk, Poland \\ grzfotyg@pg.gda.pl,nyx@eti.pg.gda.pl \\ Manuscript received April 24, 2015
}

\begin{abstract}
This paper presents a novel full-wave technique which allows for a fast 3D finite element analysis of waveguide structures containing rotatable tuning elements of arbitrary shapes. Rotation of these elements changes the resonant frequencies of the structure, which can be used in the tuning process to obtain the $S$-characteristics desired for the device. For fast commutations of the response as the tuning elements are rotated, the $3 D$ finite element method is supported by multilevel model-order reduction, orthogonal projection at the boundaries of macromodels and the operation called macromodels cloning. All the time-consuming steps are performed only once in the preparatory stage. In the tuning stage, only small parts of the domain are updated, by means of a special meshing technique. In effect, the tuning process is performed extremely rapidly. The results of the numerical experiments confirm the efficiency and validity of the proposed method.
\end{abstract}

\section{Keywords}

Finite element method (FEM), macromodels, macroelements, model-order reduction (MOR), modal projection

\section{Introduction}

Waveguide structures loaded with discontinuities, such as posts and irises, play an important role in wireless communication systems. One interesting class of such structures utilizes rotatable discontinuities, which create the desired functionalities and allow for flexible control of the frequency response of the device [1-4]. The design of these structures requires efficient optimization-oriented full-wave software that is capable of quickly updating the analysis results for arbitrary changes of the rotation angles.

The procedure for designing direct coupled-cavity waveguide filters containing half-cylinder screws using Finite Element Method (FEM) analysis has been presented in [1]. A hybrid mode-matching/boundary-contour and modematching/FEM for designing waveguide components con- taining posts and irises has been proposed in [2]. This allows for the optimization of the geometries of the waveguide filters by rotating sections, which contain posts and irises of different shapes. In [3] an efficient method based on the orthogonal expansion method and an iterative scattering procedure has been proposed for analyzing waveguide filters with rotatable posts of shapes that conform to cylindrical coordinates. An efficient 2D method combining finite difference frequency domain (FDFD) and mode-matching proposed in [4] is restricted to the posts, which are homogeneous in one direction.

In this paper, we propose a novel efficient 3D method based on FEM that, unlike [3] and [4], allows for arbitrary shapes of rotatable elements in fast tuning and optimization. It combines the meshing techniques from [5] with the model order reduction (MOR), which has been demonstrated previously for FDFD [6], [7], 2D FEM [8], [9] and 3D FEM [10], [11]. MOR is applied locally in such a way, that the subdomains of the computational domain are represented by small dense matrices, called macromodels. The mathematical formulation of this approach is similar to that presented in [10], the main difference being a new technique of incorporating the macromodels within a fixed mesh, that allows for a single macromodel to be either rotated or simultaneously rotated and replicated without the need to regenerate the mesh. This substantially speeds up the computation and makes a fast design tuning possible.

In order to allow for rotation and at the same time to perform the orthogonal projection technique of [7], [10] we developed a new set of basis functions that is applicable to cylinders. The overall cost of the MOR procedure has been significantly reduced, as the computational domain can be easily decomposed and each region can be meshed independently and the new type of macromodels can be now fully reused and cloned to be placed in different locations.

The rest of the paper is organized as follows: Section 2 provides a background of the Finite Element Method Formulation. Section 3 presents the basic steps of the approach of Model Order Reduction, which is applied locally. Section 4 
describes the technique of speeding up the reduction time by applying the orthogonal projection on macromodels' boundaries. Section 5 deals with the technique of fast analysis of the structures which contain rotatable tuning elements and it is followed by the results of the numerical examples provided in Section 6.

\section{Finite Element Method Formulation}

We consider a source-free closed 3-D region $\Omega$. The behavior of the electric field $\vec{E}$ is based on a formulation of reduced Maxwell's equations:

$$
\nabla \times \frac{1}{\mu} \nabla \times \vec{E}-\omega^{2} \epsilon \vec{E}=0
$$

where $\mu, \epsilon$ are respectively the permeability and permittivity of the medium and $\omega$ is the angular frequency. On the surfaces of perfect electric conductor $\left(\Gamma_{P E C}\right)$ and perfect magnetic conductor $\left(\Gamma_{P M C}\right)$ the following boundary conditions are applied:

$$
\begin{aligned}
& \vec{E} \times \vec{n}=0 \quad \text { on } \quad \Gamma_{\mathrm{PEC}}, \\
& \nabla \times \vec{E} \times \vec{n}=0 \quad \text { on } \Gamma_{\mathrm{PMC}} .
\end{aligned}
$$

Although the structure comprises the rotatable elements, they are used only for tuning. Therefore they do not rotate continuously and the electromagnetic analysis is performed only for stationary geometries. Applying the boundary conditions (2)-(3) to (1) and considering the $P$ ports on the surfaces $\Gamma_{i}$, one obtains the following weak formulation by means of Galerkin method [13], [15]:

$$
\begin{aligned}
\int_{\Omega}\left(\nabla \times \vec{w} \cdot \frac{1}{\mu_{r}} \nabla \times \vec{E}-k_{0}^{2} \vec{w} \cdot \epsilon_{r} \vec{E}\right) d \Omega= \\
\mathrm{j} \omega \mu_{0} \sum_{i=1}^{P} \int_{\Gamma_{i}} \vec{w} \cdot\left(\vec{n}_{i} \times \vec{H}^{i}\right) d \Gamma_{i}
\end{aligned}
$$

where $P$ is the number of the ports of the structure, $i$ is the index of the excited port $\Gamma_{i}, \vec{n}_{i}$ is a normal vector on the $i$-th port, $\vec{H}^{i}$ is the distribution of the tangential magnetic field on the $i$-th port, $\mu_{r}, \epsilon_{r}$ are relative permeability and permittivity, $k_{0}=\omega \sqrt{\mu_{0} \epsilon_{0}}$ is the wavenumber, $\vec{w}$ is a vector testing function used in the Galerkin method and $\mathrm{j}$ is the imaginary unit. The testing functions $\vec{w}$ belong to the orthogonal basis used for the expansion of the unknown vector field $\vec{E}$. In the Finite Element Method (FEM) this basis is a set of functions defined piecewise within small subregions, called finite elements, into which the whole computational domain $\Omega$ is divided by means of meshing algorithm. In the proposed approach we are using the curvilinear tetrahedral mesh generated by the Netgen software [5], [16]. Two kinds of vector basis functions are defined within each element [13], [14]. The first group is associated with six edges:

$$
\begin{aligned}
\vec{\alpha}_{i j}^{\mathrm{I}} & =\phi_{i} \nabla \phi_{j}-\phi_{j} \nabla \phi_{i}, \\
\vec{\alpha}_{i j}^{\mathrm{II}} & =\phi_{i} \nabla \phi_{j}+\phi_{j} \nabla \phi_{i},
\end{aligned}
$$

while the latter is related to four faces of tetrahedron:

$$
\begin{aligned}
& \vec{\alpha}_{i j k}^{\mathrm{III}}=3 \phi_{j} \phi_{k} \nabla \phi_{i}-\nabla\left(\phi_{i} \phi_{j} \phi_{k}\right), \\
& \vec{\alpha}_{i j k}^{\mathrm{IV}}=3 \phi_{k} \phi_{i} \nabla \phi_{j}-\nabla\left(\phi_{i} \phi_{j} \phi_{k}\right) .
\end{aligned}
$$

In (5) and (6) $\{i, j, k\} \in\{1,2,3,4\}$, and $\phi_{i}$ is the simplex coordinate associated with the $i$-th node. In fact, $\phi_{i}$ is a continues linear function, which equals one at node $i$ and zero at all other nodes. As a result, 20 basis functions up to the second-order are defined in each of the tetrahedrons.

Application of FEM basis functions (5-6) as the testing functions $\vec{w}$ and expansion of the electric field $\vec{E}$ into series of (5-6) results in the following matrix system of equations [12], [14]:

$$
\left(\mathbf{K}-k_{0}^{2} \mathbf{M}\right) \mathbf{x}=\mathbf{b},
$$

where $\mathbf{x}$ is the vector of unknown coefficients, $\mathbf{b}$ is the excitation vector, $\mathbf{K}$ and $\mathbf{M}$ are $N \times N$ stiffness and mass matrices, respectively and $N$ is the number of degrees of freedom (DOF). $\mathbf{K}$ and $\mathbf{M}$ are assembled from the local matrices $\mathbf{K}^{t}$ and $\mathbf{M}^{t}$ corresponding to each of the tetrahedrons $t$ :

$$
\begin{aligned}
K_{l k}^{t} & =\frac{1}{\mu_{r}} \int_{t} \nabla \times \vec{\alpha}_{l}^{t} \cdot \nabla \times \vec{\alpha}_{k}^{t} d \Omega, \\
M_{l k}^{t} & =\epsilon_{r} \int_{t} \vec{\alpha}_{l}^{t} \cdot \vec{\alpha}_{k}^{t} d \Omega,
\end{aligned}
$$

with $\vec{\alpha}^{t}$ being the basis functions, defined by (5) and (6), within the tetrahedron $t$ and $K_{l k}^{t}, M_{l k}^{t}$ being the elements of $\mathbf{K}^{t}$ and $\mathbf{M}^{t}$. In order to evaluate the integrals in (8) we are using the Gaussian quadrature of the tenth-order, which results in 81 quadrature points within each of the elements.

For the global problem (7) the basis functions $\vec{\alpha}^{t}$ are combined into a global set of functions $\vec{\alpha}_{n}$ and the distribution of the electric field in the whole domain $\Omega$ is approximated by means of the following expansion:

$$
\vec{E} \approx \sum_{n}^{N} x_{n} \vec{\alpha}_{n}
$$

where $x_{n}$ are the unknown coefficients in $\mathbf{x}$.

\section{Model Order Reduction}

The most time consuming step of FEM is solving the matrix system of equations of the form (7). One of the techniques to improve the efficiency of FEM is to significantly reduce the size of the system matrix by applying the Model Order Reduction (MOR) techniques. The MOR presented in this paper has been thoroughly described in [8], [10]. The main idea is to develop the appropriate transfer functions, which accurately capture the behavior of the electromagnetic field within the selected subdomains. These transfer functions are represented by so-called macromodels, which replace the original problems in 3D subdomains by the relations between the unknowns on their $2 \mathrm{D}$ boundaries. The number of these unknowns is significantly smaller than the number of variables needed to directly describe the field distribution inside the separated subdomains. 


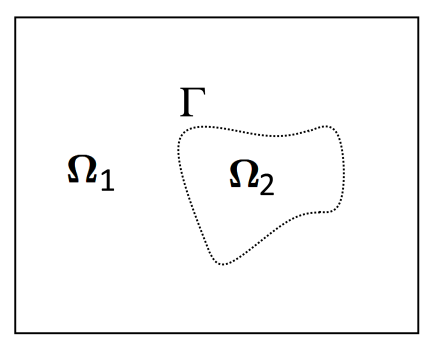

Fig. 1. A source-free 3D subdomain $\Omega_{2}$ separated from the entire domain by means of a fictitious boundary $\Gamma$.

For a single macromodel, we consider a closed sourcefree 3D subdomain $\Omega_{2}$ separated from the whole domain by a fictitious boundary $\Gamma$ (Fig. 1). It is important, that this operation does not change the original boundary conditions and thus has no impact on the solution of the electromagnetic problem.

Having consistently numbered the variables within $\Omega_{1}$, $\Gamma$ and $\Omega_{2}$, the original system (7) is split into the following blocks of matrices, which correspond to regions: $\Omega_{1}, \Gamma, \Omega_{2}$ :

$$
\begin{aligned}
&\left(\left[\begin{array}{ccc}
\mathbf{K}_{1} & \mathbf{S}_{K 1}^{T} & 0 \\
\mathbf{S}_{K 1} & \mathbf{K}_{\Gamma} & \mathbf{S}_{K 2}^{T} \\
0 & \mathbf{S}_{K 2} & \mathbf{K}_{2}
\end{array}\right]-k_{0}^{2}\left[\begin{array}{ccc}
\mathbf{M}_{1} & \mathbf{S}_{M 1}^{T} & 0 \\
\mathbf{S}_{M 1} & \mathbf{M}_{\Gamma} & \mathbf{S}_{M 2}^{T} \\
0 & \mathbf{S}_{M 2} & \mathbf{M}_{2}
\end{array}\right]\right) \\
& \cdot\left[\begin{array}{c}
\mathbf{x}_{1} \\
\mathbf{x}_{\Gamma} \\
\mathbf{x}_{2}
\end{array}\right]=\left[\begin{array}{c}
\mathbf{b}_{1} \\
0 \\
0
\end{array}\right] .
\end{aligned}
$$

Blocks $\mathbf{S}_{M}$ and $\mathbf{S}_{K}$ couple the separated subdomains, e.g. $\mathbf{S}_{K 1}$ couples regions $\Omega_{1}$ and $\Gamma$ (see $[8,10]$ for details). Note, that the excitation $\mathbf{b}_{1}$ is applied only to the outer region $\Omega_{1}$.

We assume, that the local reduction scheme will be applied to $\Omega_{2}$. By expanding the operations in (10), we obtain three matrix equations. The third o them corresponds to $\Omega_{2}$ and has the following form:

$$
\left(\mathbf{K}_{2}-k_{0}^{2} \mathbf{M}_{2}\right) \mathbf{x}_{2}=-\left(\mathbf{S}_{K 2}-k_{0}^{2} \mathbf{S}_{M 2}\right) \mathbf{x}_{\Gamma}
$$

where indices 2 and $\Gamma$ are associated with subregions: $\Omega_{2}$ and $\Gamma$, respectively and matrices $\mathbf{S}_{K 2}, \mathbf{S}_{M 2}$ couples $\Omega_{2}$ and $\Gamma$. The number of variables in $\mathbf{x}_{2}$ and $\mathbf{x}_{\Gamma}$ is denoted, as: $N_{2}$ and $N_{\Gamma}$.

The macromodel is generated using a projection basis $\mathbf{V}$ constructed by means of the Efficient Nodal Order Reduction (ENOR) algorithm [10], [17]. Basis V consists of frequencyindependent orthonormal vectors, which span the solution space in a limited frequency range. In the final step of MOR, the matrix operators of (11) related to $\Omega_{2}$ are projected onto a MOR subspace using $\mathbf{V}$. As a result, (11) is transformed into a new system:

$$
\left(\widetilde{\mathbf{K}}_{2}-k_{0}^{2} \widetilde{\mathbf{M}}_{2}\right) \widetilde{\mathbf{x}}_{2}=-\left(\widetilde{\mathbf{S}}_{K 2}-k_{0}^{2} \widetilde{\mathbf{S}}_{M 2}\right) \mathbf{x}_{\Gamma}
$$

where the tilde represents the reduction of $\mathbf{M}_{2}, \mathbf{K}_{2}, \mathbf{S}_{K 2}, \mathbf{S}_{M 2}$ and $\mathbf{x}_{2}$ :

$$
\begin{aligned}
\widetilde{\mathbf{K}}_{2} & =\mathbf{V}^{T} \mathbf{K}_{2} \mathbf{V}, \\
\widetilde{\mathbf{M}}_{2} & =\mathbf{V}^{T} \mathbf{K}_{2} \mathbf{V}, \\
\widetilde{\mathbf{x}}_{2} & =\mathbf{V}^{T} \mathbf{x}_{2}, \\
\widetilde{\mathbf{S}}_{K 2} & =\mathbf{V}^{T} \mathbf{S}_{K 2}, \\
\widetilde{\mathbf{S}}_{M 2} & =\mathbf{V}^{T} \mathbf{S}_{M 2} .
\end{aligned}
$$

Substituting (13) into (10) yields:

$$
\begin{aligned}
&\left(\left[\begin{array}{ccc}
\mathbf{K}_{1} & \mathbf{S}_{K 1}^{T} & 0 \\
\mathbf{S}_{K 1} & \mathbf{K}_{\Gamma} & \widetilde{\mathbf{S}}_{K 2}^{T} \\
0 & \widetilde{\mathbf{S}}_{K 2} & \widetilde{\mathbf{K}}_{2}
\end{array}\right]-k_{0}^{2}\left[\begin{array}{ccc}
\mathbf{M}_{1} & \mathbf{S}_{M 1}^{T} & 0 \\
\mathbf{S}_{M 1} & \mathbf{M}_{\Gamma} & \widetilde{\mathbf{S}}_{M 2}^{T} \\
0 & \widetilde{\mathbf{S}}_{M 2} & \widetilde{\mathbf{M}}_{2}
\end{array}\right]\right) \\
& \cdot\left[\begin{array}{c}
\mathbf{x}_{1} \\
\mathbf{x}_{\Gamma} \\
\widetilde{\mathbf{x}}_{2}
\end{array}\right]=\left[\begin{array}{c}
\mathbf{b}_{1} \\
0 \\
0
\end{array}\right] .
\end{aligned}
$$

The number of unknowns in $\widetilde{\mathbf{x}}_{2}$, which also represents the size of the macromodel, is much smaller than in $\mathbf{x}_{2}$, i.e.:

$$
\widetilde{N}_{2}=q N_{\Gamma} \ll N_{2},
$$

where $q$ is the reduction order. It is important to note that this MOR procedure does not introduce any frequency-dependent terms into (12), so it is performed only once for the whole frequency range for which the basis $\mathbf{V}$ has been constructed. As solving the reduced system is usually much quicker than the reduction itself, this property of MOR allows for fast frequency sweep.

In the presented example, the MOR procedure was applied for only one separated region, however for more complex problems such reduction strategy can be inefficient, since the number of variables in the selected subdomain can be too large. One of the ways to cope with this problem is to divide the computational domain into many subdomains with limited number of variables, using for example the approach presented in [18]. Subsequently, the selected subdomains are separately subject to the presented reduction scheme.

What is more, one can adopt this MOR scheme on many levels using the approach called Multilevel MOR (MMOR) [10]. On the lowest level all selected subdomains are reduced. Next, all created macromodels are grouped in such a way, that on the highest level only one macromodel (which covers the whole computational domain) remains.

\section{Orthogonal Projection on the Sub- domain Boundary}

The efficiency of MOR depends on the following factors: the reduction order $q$, the number of variables in the selected subdomain $\left(N_{2}\right)$ and the number of variables on its boundary $\left(N_{\Gamma}\right)$. To improve the efficiency of MOR, $N_{\Gamma}$ should be decreased prior to the reduction. It can be done 


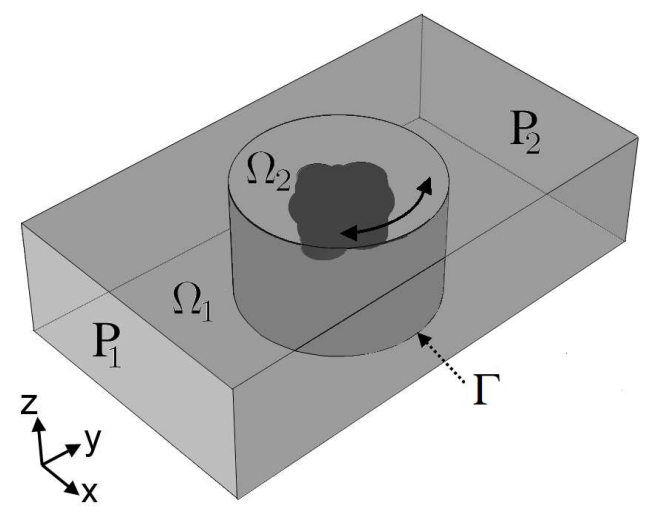

Fig. 2. A waveguide structure with an arbitrary rotatable discontinuity closed in the cylindrical subdomain $\Omega_{2}$, separated from the rest of the computational domain by means of the surface $\Gamma$. Surfaces $P_{1}$ and $P_{2}$ are the excitation ports.

through orthogonal projection of the subspace of the FEM local basis functions from the boundary $\Gamma$ onto a much smaller subspace spanned by an appropriate basis of orthogonal functions [7], [10]. In other words, the large 2D FEM basis will be replaced by the functional basis requiring much smaller number of unknown coefficients for the expansion of the field on $\Gamma$.

Let us consider a basic structure with a single arbitrary rotatable element in an empty rectangular waveguide (Fig. 2), bounded by the ports: $P_{1}$ and $P_{2}$ and the PEC walls elsewhere. We assume that this discontinuity rotates together with its nearest surrounding; it is therefore closed in the cylindrical subdomain $\Omega_{2}$ bounded by the fictitious boundary $\Gamma$. The cylinder $\Omega_{2}$ extends from the bottom to the top PEC walls of the waveguide along the $z$-axis. Although the reduction may be performed in $\Omega_{1}$ (with respect to $\Gamma, P_{1}$ and $P_{2}$ ) and $\Omega_{2}$ (with respect to $\Gamma$ ), we focus on creating a rotatable macromodel in the cylinder $\Omega_{2}$. The tangential electric field on $\Gamma$ can be approximated in the cylindrical system by means of the following series of orthogonal continuous functions:

$$
\begin{aligned}
\vec{E}_{\Gamma}(\phi, z)= & \overrightarrow{i_{z}} \sum_{i=1}^{L} \sum_{j=0}^{L} a_{i j}^{\mathrm{I}} \sin (i \phi) \cos \left(j \frac{z}{h} \pi\right)+ \\
& \overrightarrow{i_{z}} \sum_{i=0}^{L} \sum_{j=0}^{L} a_{i j}^{\mathrm{II}} \cos (i \phi) \cos \left(j \frac{z}{h} \pi\right)+ \\
& \overrightarrow{i_{\phi}} \sum_{i=0}^{L} \sum_{j=1}^{L} a_{i j}^{\mathrm{III}} \cos (i \phi) \sin \left(j \frac{z}{h} \pi\right)+ \\
& \overrightarrow{i_{\phi}} \sum_{i=1}^{L} \sum_{j=1}^{L} a_{i j}^{\mathrm{IV}} \sin (i \phi) \sin \left(j \frac{z}{h} \pi\right)
\end{aligned}
$$

where $a_{i j}^{\mathrm{I}} \ldots a_{i j}^{\mathrm{IV}}$ are the unknown amplitudes of the functions, $h$ is the height of the waveguide, $\phi \in(-\pi, \pi), z \in(0, h)$ and $L$ represent the arbitrarily chosen length of this expansion. If the field is homogeneous in the $z$ dimension, only the $E_{z}$ component of the electric field is required, thus (16) is reduced to:

$$
\vec{E}_{\Gamma}(\phi)=\overrightarrow{i_{z}} \sum_{i=1}^{L} a_{i}^{\mathrm{I}} \sin (i \phi)+\overrightarrow{i_{z}} \sum_{i=0}^{L} a_{i}^{\mathrm{II}} \cos (i \phi) .
$$

The series (16) and (17) define the basis functions to be used in the orthogonal projection mentioned in the beginning of this section. It can be expressed as a linear combination of $N_{\Gamma}^{\prime}$ functions in the following compact form:

$$
\vec{E}_{\Gamma}(\phi, z)=\left[\vec{e}_{\Gamma}^{1}(\phi, z), \vec{e}_{\Gamma}^{2}(\phi, z) \ldots \vec{e}_{\Gamma}^{N_{\Gamma}^{\prime}}(\phi, z)\right] \mathbf{a},
$$

where $\mathbf{a}$ is the vector of unknown amplitudes and the number of functions is:

$$
N_{\Gamma}^{\prime}=(2 L+1)^{2}
$$

for series (16) and:

$$
N_{\Gamma}^{\prime}=2 L+1
$$

for series (17).

The continuous functions from (18) are discretized by projecting them onto the subspace spanned on the basis functions defined by (5) and (6), which are associated with the edges and facets on $\Gamma$. As a result, the following orthogonal basis with $N_{\Gamma}^{\prime}$ vectors arises:

$$
\mathbf{E}_{\Gamma}=\left[\mathbf{e}_{\Gamma}^{1}, \mathbf{e}_{\Gamma}^{2} \ldots \mathbf{e}_{\Gamma}^{N^{\prime}{ }_{\Gamma}}\right]
$$

The vector $\mathbf{e}_{\Gamma}^{k}$ is computed as follows:

$$
\mathbf{e}_{\Gamma}^{k}=\mathbf{M}_{\Gamma}^{-1} \mathbf{u}
$$

where the $n$-th element of $\mathbf{u}$ has the form:

$$
u_{n}=\int_{\Gamma} \vec{\alpha}_{n} \cdot \vec{e}_{\Gamma}^{k}(\phi, z) d \Gamma .
$$

$\mathbf{M}_{\Gamma}$ represents the mass matrix associated with the basis functions (5)-(6) at the boundary $\Gamma$.

Subsequently, the variables from FEM system corresponding to the regions $\Omega_{2}$ and $\Gamma$ are projected onto the subspace spanned by (21). The resulting system is derived from the initial FEM system (11) and can be written in the compact form:

$$
\left(\mathbf{K}_{2}-k_{0}^{2} \mathbf{M}_{2}\right) \mathbf{x}_{2}=-\left(\mathbf{S}^{\prime}{ }_{K 2}-k_{0}^{2} \mathbf{S}^{\prime}{ }_{M 2}\right) \mathbf{x}_{\Gamma}^{\prime}
$$

where the prime sign refers to the results of the projection:

$$
\begin{aligned}
\mathbf{S}_{M 2}^{\prime} & =\mathbf{S}_{M 2} \mathbf{E}_{\Gamma}, \\
\mathbf{S}^{\prime}{ }_{K 2} & =\mathbf{S}_{K 2} \mathbf{E}_{\Gamma}, \\
\mathbf{x}_{\Gamma}^{\prime} & =\mathbf{x}_{\Gamma} \mathbf{E}_{\Gamma} .
\end{aligned}
$$

For the system (24), the subsequent model-order reduction is performed according to the same procedure as the one presented for the system (11). Owing to the orthogonal projection, the number of variables on the boundary of the macromodel subdomain is significantly reduced $\left(N_{\Gamma}^{\prime} \ll N_{\Gamma}\right)$. Accordingly, the size of the resulting macromodel $\widetilde{N}_{2}^{\prime} \times \widetilde{N}_{2}^{\prime}$ is decreased to the same extent, since: 


$$
\begin{aligned}
& \widetilde{N}_{2}^{\prime}=q N_{\Gamma}^{\prime} \ll \widetilde{N}_{2}, \\
& \widetilde{N}_{2}=q N_{\Gamma} .
\end{aligned}
$$

The same orthogonal projection and MOR procedure may also be applied to the subdomain $\Omega_{1}$. However, on the boundaries $P_{1}$ and $P_{2}$, the modal expansion of the waveguide TE and TM modes is the natural choice for the projection [10].

\section{Analysis of Structures Containing Rotatable Elements}

Without loss of generality, we refer our analysis to waveguide filters containing rotatable tuning elements, which will be presented for a section with a single rotatable discontinuity, such as the one shown in Fig. 3. The standard approach to FEM analysis of structures where the discontinuity rotates, requires regeneration of the mesh and of the corresponding FEM matrices in the entire structure after each rotation step.

We propose a method that allows the inner $\left(\Omega_{3}\right)$ and outer $\left(\Omega_{1}\right)$ domain to be meshed only once. The inner and outer regions are then processed by MOR (see section 3 ). If the analyzed structure contains many identical tuning elements, one can use a single macromodel to represent all these subregions; this operation is called macromodels cloning. Next, the two macromodels are coalesced via a narrow buffer layer $\Omega_{2}$, which is updated as the inner region rotates.

The proposed procedure is performed as a preparatory stage followed by a tuning stage. The first of these involves all the operations that can be performed in the subdomains of constant mesh - such as generating the mesh, the FEM matrices, and macromodels - while in the second stage, only the buffer is updated and the final system of equations is solved.

\subsection{Preparatory Stage}

This stage is the most time-consuming part of the entire analysis, but its operations are carried out only once. After generating the mesh in the separate $\Omega_{1}$ and $\Omega_{3}$ subdomains, orthogonal projection is performed on their boundaries: $\Gamma_{2}$ for $\Omega_{3}$ and $\left(\Gamma_{1}, P_{1}, P_{2}\right)$ for $\Omega_{1}$. MOR is performed with respect to these compressed boundaries, and so the macromodels are created separately in $\Omega_{1}$ and $\Omega_{3}$. These macromodels are combined in the matrices of the final system of equations, from which only a small part (corresponding to the buffer) is missing. Such constant templates for the system matrices will be preserved for all subsequent tuning stages. The stiffness matrices of these two subregions have the following form:

$$
\mathbf{K}_{\mathrm{I}}=\left[\begin{array}{ccc}
\mathbf{K}_{P}^{\prime} & \widetilde{\mathbf{S}}_{P}^{T} & 0 \\
\widetilde{\mathbf{S}^{\prime}} & \widetilde{\mathbf{K}}_{\Omega 1} & \widetilde{\mathbf{S}}_{\Omega 1}^{T} \\
0 & \widetilde{\mathbf{S}}_{\Omega 1}^{\prime} & \mathbf{K}_{\Gamma 1 A}^{\prime}
\end{array}\right],
$$

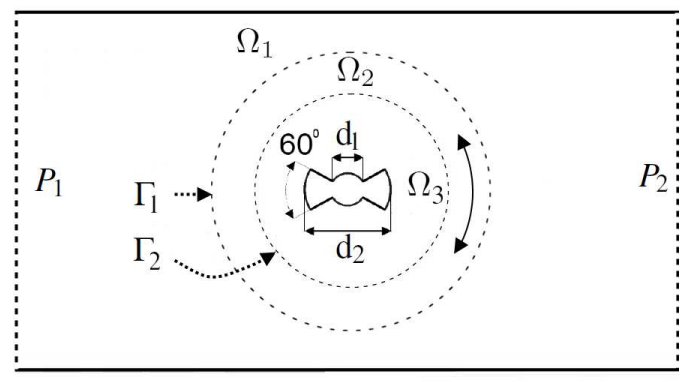

Fig. 3. Elevation view of the $3 \mathrm{D}$ WR- 90 waveguide structure with centrally placed rotatable bow-tie tuning element. $d_{1}=1.5 \mathrm{~mm}, d_{2}=5 \mathrm{~mm}$, length $=40 \mathrm{~mm}$, width $=22.86 \mathrm{~mm}$, height of the waveguide $=10.16 \mathrm{~mm}$, height of the bow-tie $=9.16 \mathrm{~mm}$, diameter of $\Gamma_{1}=9 \mathrm{~mm}$ and diameter of $\Gamma_{2}=8 \mathrm{~mm}$, rotation angle of $\Omega_{3}: \phi=0$.

$$
\mathbf{K}_{\mathrm{III}}=\left[\begin{array}{cc}
\mathbf{K}_{\Gamma 2 B}^{\prime} & \widetilde{\mathbf{S}}_{\Omega 3}^{T} \\
{\widetilde{\mathbf{S}^{\prime}}}_{\Omega 3} & \widetilde{\mathbf{K}}_{\Omega 3}
\end{array}\right] .
$$

The mass matrices have the same structure, therefore they are derived following the same procedure.

\subsection{Tuning Stage}

The tuning stage is performed repeatedly for each rotation angle, but involves only the least time-consuming operations. Having set the rotation angle, the new mesh and the corresponding FEM matrices of the buffer $\Omega_{2}$ are generated, based on the surface mesh of $\Gamma_{1}$ and $\Gamma_{2}$ [5]. In order to reflect the rotation of $\Omega_{3}$ in the updated buffer mesh, only the surface mesh of $\Gamma_{2}$ needs to be rotated. Next, the matrices associated with the buffer are generated in the following form:

$$
\mathbf{K}_{\mathrm{II}}=\left[\begin{array}{ccc}
\mathbf{K}_{\Gamma 1 B}^{\prime} & \widetilde{\mathbf{S}}_{\Gamma 1}^{T} & 0 \\
\widetilde{\mathbf{S}}_{\Gamma 1}^{\prime} & \mathbf{K}_{\Omega 2} & \widetilde{\mathbf{S}}_{\Gamma 2}^{T} \\
0 & \widetilde{\mathbf{S}}_{\Gamma 2}^{\prime} & \mathbf{K}_{\Gamma 2 A}^{\prime}
\end{array}\right] .
$$

Then they are embedded in the templates generated in the preparatory stage, which connects the macromodels representing $\Omega_{1}$ and $\Omega_{3}$, by adding matrices $\mathbf{K}_{\mathrm{I}}, \mathbf{K}_{\mathrm{II}}$ and $\mathbf{K}_{\mathrm{III}}$ :

$$
\mathbf{K}=\left[\begin{array}{cccccc}
\mathbf{K}_{P}^{\prime} & \widetilde{\mathbf{S}}_{P}^{T} & 0 & 0 & 0 & 0 \\
\widetilde{\mathbf{S}}_{P}^{\prime} & \widetilde{\mathbf{K}}_{\Omega 1}^{T} & \widetilde{\mathbf{S}}_{\Omega 1}^{T} & 0 & 0 & 0 \\
0 & \widetilde{\mathbf{S}}_{\Omega 1}^{\prime} & \mathbf{K}_{\Gamma 1 A}^{\prime}+\mathbf{K}_{\Gamma 1 B}^{\prime} & \widetilde{\mathbf{S}}_{\Gamma 1}^{T} & 0 & 0 \\
0 & 0 & \widetilde{\mathbf{S}}_{\Gamma 1}^{\prime} & \mathbf{K}_{\Omega 2}^{\prime} & {\widetilde{\mathbf{S}^{\prime}}}_{\Gamma 2}^{T} & 0 \\
0 & 0 & 0 & \widetilde{\mathbf{S}}_{\Gamma 2}^{\prime} & \mathbf{K}_{\Gamma 2 A}^{\prime}+\mathbf{K}_{\Gamma 2 B}^{\prime} & \widetilde{\mathbf{S}}_{\Omega 3}^{T} \\
0 & 0 & 0 & 0 & \widetilde{\mathbf{S}}_{\Omega 3}^{\prime} & \widetilde{\mathbf{K}}_{\Omega 3}
\end{array}\right] .
$$

Now the optional second model-order reduction may be applied to the whole structure with respect to the ports $P_{1}$ and $P_{2}$, represented by the matrices $\mathbf{K}_{P}^{\prime}$ and $\mathbf{M}_{P}^{\prime}$. Since the resulting matrices have been significantly reduced, the computational time needed to solve the final system of equations is negligible [10]. 


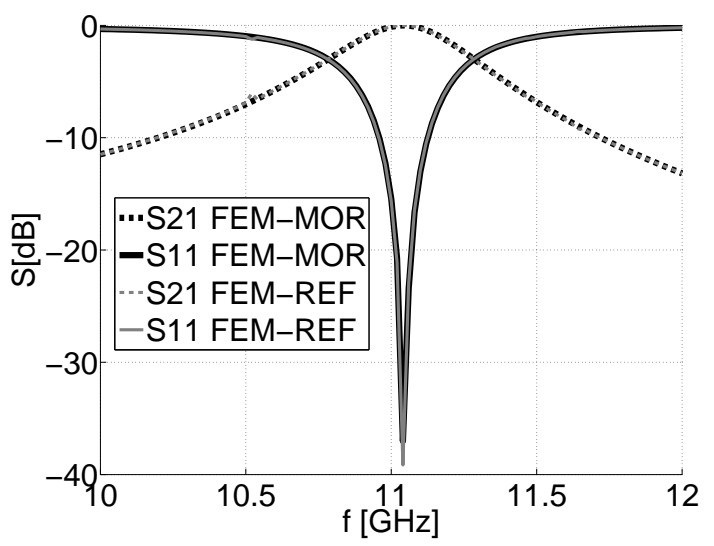

Fig. 4. $S$-characteristics of the structure obtained using the standard FEM and FEM-MOR for $\phi=30$

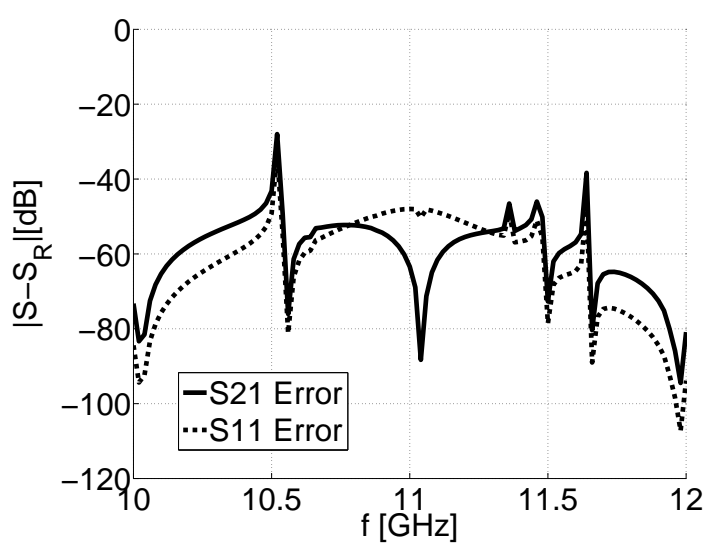

Fig. 5. Absolute error for $S_{11}$ and $S_{21}$ values computed using a FEM and FEM-MOR.

\section{Numerical Examples}

All the numerical examples presented in this section were calculated on a computer with an Intel i7 processor and 16 GB RAM in MATLAB. We first consider a structure with a single bow-tie discontinuity that does not span the waveguide height (Fig. 3). The aim of the analysis was to compute the $S$-parameters in the bandwidth of 10-12 GHz, assuming $T E_{10}$ mode excitation. First, in order to generate the reference results, the structure was analyzed by means of the standard FEM formulation using 52,200 degrees of freedom $(\mathrm{DoF})$. It took $1.5 \mathrm{~s}$ to generate a tetrahedral mesh, $37.9 \mathrm{~s}$ to assemble the matrices, and $132.6 \mathrm{~s}$ to compute the whole 51 frequency point $(f p)$ characteristic - in total, $170.5 \mathrm{~s}$ (Tab. 1, example I). Next, the structure was simulated using the proposed method. It took $1.5 \mathrm{~s}$ and $38.3 \mathrm{~s}$ to obtain the mesh and corresponding matrices, respectively, resulting in 58,002 DoF. We then projected the basis functions for $\Gamma_{1}$ and $\Gamma_{2}$ onto a subspace spanned by 49 functions (series (16) with $L=3$ ), and the basis functions from $P_{1}$ and $P_{2}$ onto a subspace spanned by the dominant waveguide mode $T E_{10}$. The preparatory stage was completed by the reduction of the subdomains: $\Omega_{1}$ (reduction order $\left.q=4,10.5 \mathrm{~s}\right)$ and $\Omega_{3}(q=2$ and $5.2 \mathrm{~s}$ ). The total time for computation of the preparatory

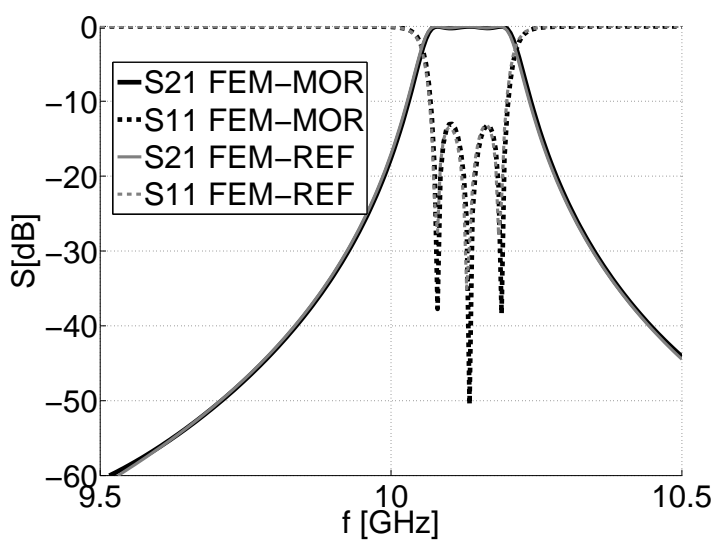

Fig. 6. $S$-characteristics of the waveguide filter with inhomogeneity in the $z$ direction obtained using standard FEM and FEM-MOR for $\phi_{1 \ldots 4}=\left[-30^{\circ},-28^{\circ}, 28^{\circ}, 30^{\circ}\right]$.

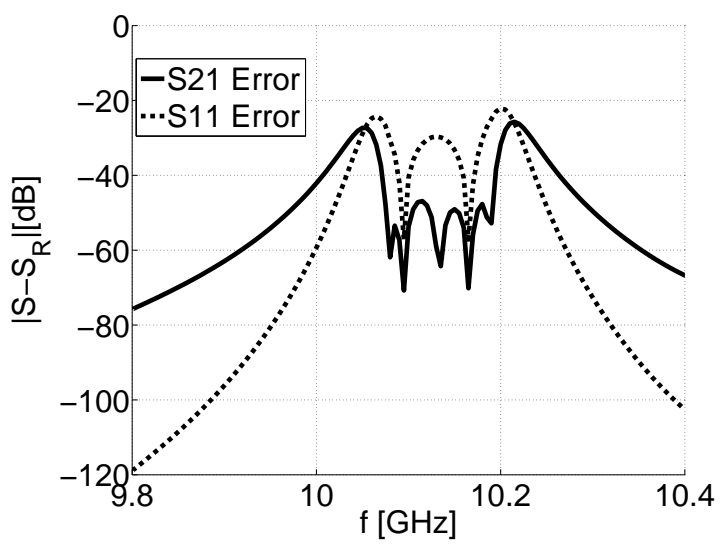

Fig. 7. Absolute error for $S_{11}$ and $S_{21}$ values computed using a FEM and FEM-MOR for $\phi_{1 \ldots 4}=$ $\left[-30^{\circ},-28^{\circ}, 28^{\circ}, 30^{\circ}\right]$.

stage was $61 \mathrm{~s}$; however, it was performed only once for all subsequent tuning stages. Each tuning stage, which includes the second reduction of the entire domain $(q=6)$ and the final solution in the whole frequency band, takes only $7.4 \mathrm{~s}$ (Tab. 1). The $S$-characteristics of the structure obtained by means of the standard FEM and FEM-MOR for $\phi=30$ are presented in Fig. 4 and the error plots are shown in Fig. 5. Very good agreement can be observed over the entire bandwidth.

In the standard approach the whole FEM procedure has to be repeated each time the geometry changes (e.g. for 10 tuning stages the overall standard FEM time is $10 \times 170.5 \mathrm{~s}=1705 \mathrm{~s}$ ). In our approach the preparatory stage is performed once and only the tuning stage is performed after each of the geometry changes $(61 \mathrm{~s}+10 \times 7.4 \mathrm{~s}=135 \mathrm{~s})$. Therefore, the speed-up of the FEM-MOR compared to the standard FEM increases with the number of tuning stages. For 1 and 10 tuning stages the FEM-MOR is 2.4 and 12.4 times faster, respectively. Since the choice of $L$ affects only the preparatory stage, it has quite moderate influence on the overall CPU time. For $\mathrm{L}=4$ the speed-up for 10 tunings decreases from 12.6 to 11.6 (only by $8 \%$ ). 


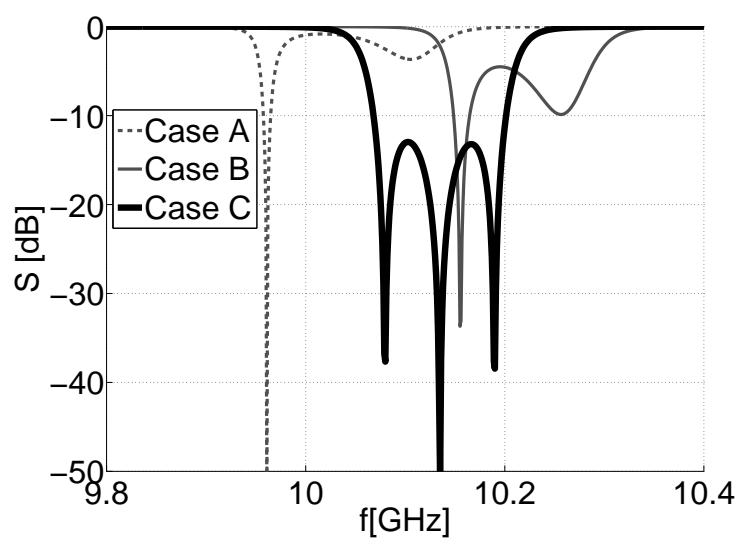

Fig. 8. $S_{11}$-characteristics of the waveguide filter obtained using FEM-MOR for 3 cases of $\phi_{1} \ldots 4$ : $A\left[-10^{\circ},-80^{\circ}, 80^{\circ}, 10^{\circ}\right], B\left[50^{\circ}, 0^{\circ}, 0^{\circ},-50^{\circ}\right] \quad$ and $C\left[-30^{\circ},-28^{\circ}, 28^{\circ}, 30^{\circ}\right]$.

\begin{tabular}{|l|l|c|r|c|}
\hline Example & standard & \multicolumn{2}{|c|}{ FEM-MOR stages } & speedup for \\
\cline { 3 - 4 } & FEM & preparatory & tuning & 10 tunings \\
\hline I & $170.5 \mathrm{~s}$ & $61.0 \mathrm{~s}$ & $7.4 \mathrm{~s}$ & 12.6 \\
\hline II & $986.5 \mathrm{~s}$ & $152.9 \mathrm{~s}$ & $23.9 \mathrm{~s}$ & 25.2 \\
\hline III & $894.3 \mathrm{~s}$ & $89.6 \mathrm{~s}$ & $21.8 \mathrm{~s}$ & 29.0 \\
\hline
\end{tabular}

Tab. 1. Comparison of the performance of FEM and FEMMOR.

The second example deals with a third-order waveguide filter containing four metallic elliptical posts with the passband at 10.05-10.2 GHz. The geometry and dimensions of the filter are provided in [4], where the posts are full-height while in our case they are partial-height $(h=9.16 \mathrm{~mm})$. Therefore, we refer our results to standard FEM instead of [4]. Each of the rotatable elements is enclosed in cylindrical subdomains of diameter $5 \mathrm{~mm}$ with buffer layers $1 \mathrm{~mm}$ thick. As in the previous example $L$ is set to 3 . In the reduction process, we used $q=2$ for each of the cylindrical subdomains and $q=4$ for the outer region. In the tuning stage 4 values of $\phi_{1 \ldots 4}$ are set, and 4 corresponding buffer meshes and matrices are generated. The second reduction (with respect to the ports of the structure) using $q=6$ is applied, and the resulting system of equations is solved in $51 \mathrm{fp}$. The computation times are compared in Tab. 1. Figures 6 and 7 show respectively the $S$-characteristics of the filter obtained by standard FEM and FEM-MOR and the error plots. Figures 8 and 9 show the characteristics for different values of the rotation angles $\phi_{1} \ldots 4$. The computation times for the tuning stages do not depend on the rotation angles. For 10 tuning stages, the computation time is as much as 25 times shorter.

The final test (example III) involves the same waveguide filter as in the example II, but for the full height of the elliptical cylinders. Since the structure is homogeneous in the $z$ dimension, only the $E_{z}$ component of the electric field is nonzero. In the orthogonal projection, we therefore used the expansion (17). In effect, the number of functions in the projection basis is 7 (series (17) with $L=3$ ), compared to 49 in example II. The other parameters of the FEM-MOR

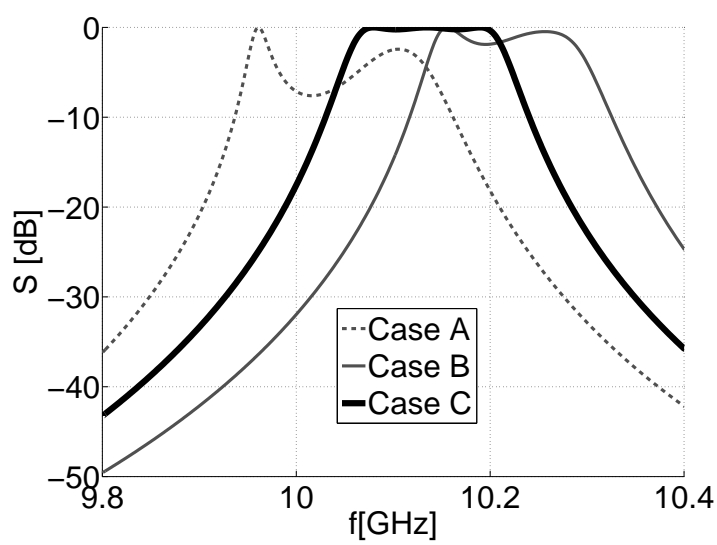

Fig. 9. $S_{21}$-characteristics of the waveguide filter obtained using FEM-MOR for 3 cases of $\phi_{1 \ldots 4}$ : $A\left[-10^{\circ},-80^{\circ}, 80^{\circ}, 10^{\circ}\right], B\left[50^{\circ}, 0^{\circ}, 0^{\circ},-50^{\circ}\right] \quad$ and $C\left[-30^{\circ},-28^{\circ}, 28^{\circ}, 30^{\circ}\right]$.

analysis remain unchanged. The simulation times for FEM and FEM-MOR are presented in Tab. 1 (example III). The homogeneity of the tuning posts reduces mostly the time for the preparatory stages. The tuning times remain comparable for both full and partial height cylinders.

\section{Conclusion}

In this paper, we presented a technique for the efficient full-wave analysis of structures that contain rotatable tuning elements. The 3D FEM method, chosen to allow arbitrary geometries of the structure and rotating elements, is enhanced by model order reduction (MOR). In order to speed up analysis, the most time-consuming operations - mesh generation, FEM matrix assembly, orthogonal projection, and MOR - are grouped and performed only once prior the subsequent massively repeated tuning steps. The rotations of the tuning elements require only minor updates in tiny subdomains, which involves operations that can be performed very quickly. It was demonstrated through numerical examples that the presented technique significantly accelerates the analysis, and can be further developed into a versatile optimization tool.

\section{Acknowledgments}

This work was supported by the Polish National Science Center under Contract PRO-2013/09/N/ST7/02268.

\section{References}

[1] COCCIOLI, R., MORINI, A., PELOSI, G., et al. Design of tolerance-corrected filters employing half-cylinder posts. IEEE Transactions on Microwave Theory and Techniques, 1998, vol. 46, no. 1, p. 116-118. ISSN: 0018-9480. DOI: $10.1109 / 22.654930$

[2] ARNDT, F., BEYER, R., REITER, J. M., et al. Automated design of waveguide components using hybrid mode-matching/numerical EM building-blocks in optimization-oriented CAD frameworksstate of the art and recent advances. IEEE Transactions on Microwave Theory and Techniques, 1997, vol. 45, no. 5, p. 747-760. ISSN: 0018-9480. DOI: 10.1109/22.575597 
[3] LECH, R., MAZUR, J. Tunable waveguide filter with bow-tie metallic posts. IEE Proceedings - Microwaves, Antennas and Propagation, 2004, vol. 151, no. 2, p. 156-160. ISSN 1359-706X. DOI: 10.1049/ip-map:20040166

[4] KUSIEK, A., LECH, R., MAZUR, J. A new hybrid method for analysis of scattering from arbitrary configuration of cylindrical objects. IEEE Transactions on Antennas and Propagation, 2008, vol. 56, no. 6 , p. 1725-1733. ISSN: 0018-926X. DOI: 10.1109/TAP.2008.923339

[5] FOTYGA, G., BIELSKI, P., NYKA, K. A 3D-FEM mesh technique for fast analysis of waveguide problems containing rotatable tuning elements. In 20th International Conference on Microwaves, Radar and Wireless Communication (MIKON), 2014, June 2014, p. 1-4. ISBN: 978-617-607-553-0. DOI: 10.1109/MIKON.2014.6899875

[6] KULAS, L., MROZOWSKI, M. Multilevel model order reduction. IEEE Microwave and Wireless Components Letters, 2004, vol. 14, no. 4, p. 165-167. ISSN: 1531-1309. DOI: 10.1109/LMWC.2004.827113

[7] KULAS, L., KOWALCZYK, P., MROZOWSKI, M. A novel modal technique for time and frequency domain analysis of waveguide components. IEEE Microwave and Wireless Components Letters, 2011, vol. 21, no. 1, p. 7-9. ISSN: 1531-1309. DOI: 10.1109/LMWC.2010.2089439

[8] FOTYGA, G., NYKA, K., KULAS, L. A new type of macroelements for efficient two-dimensional FEM analysis. IEEE Antennas and Wireless Propagation Letters, 2011, vol. 10, p. 270-273. ISSN: 1536-1225. DOI: 10.1109/LAWP.2011.2134063

[9] ZHU, Y., CANGELLARIS, A. C. Macro-elements for efficient FEM simulation of small geometric features in waveguide components. IEEE Transactions on Microwave Theory and Techniques, 2000, vol. 48, no. 12, p. 2254-2260. ISSN: 0018-9480. DOI: $10.1109 / 22.898972$

[10] FOTYGA, G., NYKA, K., MROZOWSKI, M. Multilevel model order reduction with generalized compression of boundaries for 3-D FEM electromagnetic analysis. Progress In Electromagnetics Research, 2013, vol. 139, p. 743-759. ISSN: 1070-4698. DOI: $10.2528 /$ PIER13032708.

[11] SCHEIBER, C., SCHULTSCHIK, A., BIRO, O., et al. A model order reduction method for efficient band structure calculations of photonic crystals. IEEE Transactions on Magnetics, 2011, vol. 47, no. 5, p. 1534-1537. ISSN: 0018-9464. DOI: 10.1109/TMAG.2010.2081973

[12] PELOSI, G., COCCIOLI, R., SELLERI, S. Quick Finite Elements for Electromagnetic Waves. 2nd ed. Norwood: Artech House, 2009. ISBN: 978-1-59693-345-3
[13] INGELSTROM, P. A new set of H (curl)-conforming hierarchical basis functions for tetrahedral meshes. IEEE Transactions on Microwave Theory and Techniques, 2006, vol. 54, no. 1, p. 106-114. ISSN: 0018-9480. DOI: 10.1109/TMTT.2005.860295

[14] LAMECKI, A. BALEWSKI, L. MROZOWSKI, M. An efficient framework for fast computer aided design of microwave circuits based on the higher-order 3D finite-element method. Radioengineering, 2014, vol. 23, no. 4, p. 970-978. ISSN: 1805-9600

[15] RUBIO, J., ARROYO, J., ZAPATA, J. Analysis of passive microwave circuits by using a hybrid 2-D and 3-D finite-element modematching method. IEEE Transactions on Microwave Theory and Techniques, 1999, vol. 47, no. 9, p. 1746-1749. ISSN: 0018-9480. DOI: $10.1109 / 22.788618$

[16] SCHÖBERL, J. NETGEN An advancing front 2D/3D-mesh generator based on abstract rules. Computing and Visualization in Science, 1997, vol. 1, no. 1, p. 41-52. ISSN: 1432-9360. DOI: $10.1007 / \mathrm{s} 007910050004$

[17] SHEEHAN, B. N. ENOR: Model order reduction of RLC circuits using nodal equations for efficient factorization. In Proceedings of the 36th Design Automation Conference. New Orleans, June 1999, p. 17-21. ISBN: 1-58113-109-7. DOI: $10.1145 / 309847.309855$

[18] SHAO, Y., PENG, Z., LEE, J.-F. Full-wave real-life 3-D package signal integrity analysis using nonconformal domain decomposition method. IEEE Transactions on Microwave Theory and Techniques, 2011, vol. 59, no. 2, p. 230-241. ISSN: 0018-9480. DOI: 10.1109/TMTT.2010.2095876

\section{About the Authors...}

Grzegorz FOTYGA was born in Gdansk, Poland. He received his M.Sc. Electrical Engineering degree from Gdansk University of Technology in 2009 . He is currently working toward Ph.D. in the area of Model Order Reduction in Finite Element Method.

Krzysztof NYKA was born in Gdynia, Poland. He received his M.Sc. and Ph.D. degrees from Gdansk University of Technology. He is currently an assistant professor with the Department of Microwave and Antenna Engineering, Gdansk University of Technology. His research interests include computational electrodynamics, especially the application of FEM and MOR, as well as microwave circuits for wireless systems. 